\title{
PG 0844+349 revisited - is there any outflow?
}

\author{
W. Brinkmann ${ }^{1}$, T. Wang ${ }^{2}$, D. Grupe ${ }^{3}$, and C. Raeth ${ }^{1}$ \\ 1 Max-Planck-Institut für Extraterrestrische Physik, Postfach 1312, 85741 Garching, Germany \\ e-mail: wpb@mpe.mpg.de \\ 2 University of Science and Technology of China, HeFei, Anhui 230026, PR China \\ e-mail: twang@ustc. edu.cn \\ 3 Astronomy Department, Pennsylvania State University, 525 Davey Lab, University Park, PA 16802, USA \\ e-mail: grupe@astro.psu.edu
}

Received 4 July 2005 / Accepted 29 November 2005

\section{ABSTRACT}

Aims. The detection of high velocity absorption lines from highly ionized material has been reported recently from the X-ray spectrum of the narrow line Seyfert 1 galaxy PG 0844+349 and were interpreted as a relativistic outflow from the source. We studied this outflow because it would have important implications for our understanding of narrow line Seyfert 1 galaxies.

Methods. To address the reality of these absorption features, we analyzed a long XMM observation with the MOS cameras on this object. Results. We did not detect the absorption lines claimed, and a re-analysis of the previous data set with the most recent detector calibrations did not confirm the earlier results. The X-ray spectra can be well modeled with a power law plus a bremsstrahlung component. With this description, the soft and the harder $2-10 \mathrm{keV} \mathrm{X-ray} \mathrm{flux} \mathrm{changed} \mathrm{by} \mathrm{a} \mathrm{similar} \mathrm{fraction}(\simeq 25 \%)$ between the two observations with only small changes in the spectral form. An analysis of the hardness ratio variations within a single observation does not show any strong correlation between the hardness ratio and the continuum luminosity and we do not detect substantial lags between the hard and soft band fluxes.

Key words. galaxies: active - quasars: general - quasars: individual: PG 0844+349

\section{Introduction}

In a recent paper Pounds et al. (2003a) reported the discovery of absorption structures in the X-ray spectrum of the narrow emission line quasar PG 1211+143, which were interpreted as originating from a fast, highly ionized outflow from the object. If this interpretation is correct it would have important consequences for our understanding of quasars. The matter with high column densities of $\sim 5 \times 10^{23} \mathrm{~cm}^{-2}$ and inferred outflow velocities of $\sim 0.08 \mathrm{c}$ would carry a substantial fraction of the available accretion energy. Due to the high column density, the inner flow is likely to be optically thick, thus providing a natural explanation for the big blue bump and the soft X-ray emission of the source.

A highly blue-shifted ionized Fe $\mathrm{K}$ edge was discovered in the spectrum of the high- $z$ BALQSO APM $08279+5255$ in a long XMM-Newton observation by Hasinger et al. (2002), while Chartas et al. (2002) claim detection of two broad absorption features from Chandra observations of the source. Assuming that the absorption lines are from Fe XXV $\mathrm{K} \alpha$ outflow, velocities of $\sim 0.2 \mathrm{c}$ and $\sim 0.4 \mathrm{c}$ were inferred. The model adopted in the analysis of the XMM data does not provide an acceptable fit to the Chandra data and might indicate considerable spectral time variability. Indications of out-flowing matter at velocities of $\sim 0.1 \mathrm{c}$ and $\sim 0.34 \mathrm{c}$ were further seen in an XMM-Newton observation of another BAL quasar, PG 1115+080 (Chartas et al. 2003). The deduced properties of the high-energy (2-5 keV band) and the low-energy absorbers (0.2-0.6 keV band) indicate that these absorbers are very likely distinct. In both BAL objects the fluxes are greatly magnified by gravitational lensing, and significant variability seems to be present in the sources. However, the data quality is rather limited for these high red-shift sources.

A second case for outflow from a narrow line Seyfert galaxy (NLS1) has been claimed by Pounds et al. (2003b) for PG $0844+349$ from an observation of about $20 \mathrm{ks}$ by XMM-Newton. The absorption features in the $~ 9-13 \AA$ band, as well as at $\sim 3.0 \mathrm{keV}$ and $\sim 8.2 \mathrm{keV}$, were not seen in the original analysis of the data by Brinkmann et al. (2003); however, different versions of instrument responses had been used.

Recently, Dasgupta et al. (2004) reported the discovery of analogous features in the XMM spectrum of the narrow emission line quasar PG 1404+226. Similar results for this object and two other NLS1 galaxies, 1H 0707-495 and IRAS 13224-3809, were presented earlier from ASCA 
observations by Leighly et al. (1997). However, the spectra are generally of limited quality and the significances of the features are comparatively low.

Apart from the low red-shift quasar PG 1211+143, identifications of blue-shifted, narrow absorption lines, as well as their statistical significances, are somewhat uncertain. However, McKernan et al. (2004) point out that part of the absorption attributed to warm $\mathrm{Fe}-K$ band absorption intrinsic to PG 1211+143 might in fact be due to OVII and OVIII absorption of hot, local gas. And, more recently, in a re-analysis of the XMM-Newton data, based on an alternative interpretation of the data, Kaspi \& Behar (2005) find much lower outflow velocities in PG 1211+143, similar to those commonly observed in Seyfert galaxies. As the existence of quasar outflows is of outmost importance for the understanding of these objects, in particular for the NLS1 galaxies, more high quality data and the definite confirmation of candidate objects is required.

One of these candidates is PG $0844+349$, for which only one relatively short XMM-Newton observation has existed so far, and no unequivocal results can be claimed from it. It is a nearby $(z=0.064)$ bright object accreting near its Eddington rate. In the next section we first re-analyze its data from the observation of November 2000, focusing on the absorption features. We then present an analysis of the MOS data of the source when it was found serendipitously in the field of view of a $\sim 86$ ks observation of the cluster of galaxies Vik 59 (Vikhlinin et al. 1998) in October 2001.

\section{Observations and data reduction}

The XMM-Newton observations of PG 0844+349 from November 5, 2000 were first presented by Brinkmann et al. (2003). The total exposure time is $\sim 23 \mathrm{ks}$, but the background count rate was high during a large fraction of the observation, leading to a substantial reduction of the usable exposure. We reprocessed the data with XMMSAS version 6.1. and applied for the analysis the most recent calibration files as of December 12, 2004.

With a count rate of $\gtrsim 7 \mathrm{cts}^{-1}$ in the high state, the PN detector, which was operated in Full Window mode, showed strong indications of pile-up, clearly apparent from the XMMSAS task epatplot. We therefore disregarded the innermost $3 \times 3$ RAW pixels (one RAW pixel $\sim 4.1^{\prime \prime}$ ) from the center of the point spread function for the spectral analysis, which does not introduce any noticeable spectral distortions (Aschenbach 2001), and confirmed that pile-up is then negligible. We further excluded time intervals with high background (count rate above $10 \mathrm{keV}$ larger than $2 \mathrm{cts} \mathrm{s}^{-1}$ ), which left us with an exposure for the spectral analysis of $\sim 12670 \mathrm{~s}$ and a total of $\sim 47000$ photons in the $0.3-10 \mathrm{keV}$ energy band. For the spectral fits we selected single and double events (PATTERN $\leq$ 4 and FLAG $=0$ ) from a region of $27 \times 26$ RAW pixels around the source position; for details of the instruments see Ehle et al. (2001).

For the MOS data, pile-up was negligible. The source counts were accumulated within a circle of radius $45^{\prime \prime}$ surrounding the position of PG $0844+349$ and the background data were extracted from a similar region close to the source.
Spectral responses and effective area were generated with rmfgen, and arfgen and photons with PATTERN $\leq 12$ and $\mathrm{FLAG}=0$ were used. During the net exposure, about 23000 photons were accumulated for each MOS camera for the spectral fits.

The total exposure for the observation of Vik 59 in October 2001 is $86 \mathrm{ks}$. The source is offset from the center of the FOV by $\sim 6$ ' 8 . The two MOS cameras were in Primary Full Window mode with a thin filter. During the whole observation, the background rate was low, amounting only to $\lesssim 1.5 \%$ of the source count rate. The data during two smaller, short flares and the last $\sim 2.5$ ks of the observation, where the background strongly increased, were disregarded from the analysis. As above, the data were reprocessed, responses were generated with the newest XMMSAS, and for the spectral analysis the same data selection criteria were applied. The net exposures of each MOS camera after GTI (Good Time Interval) selection was $\sim 77 \mathrm{ks}$; the average count rate for the screened $0.3-10 \mathrm{keV}$ data for the MOS1 is $1.41 \mathrm{cts} / \mathrm{s}$, and for the MOS2 $1.40 \mathrm{cts} / \mathrm{s}$.

\section{Results}

\subsection{Spectral modeling}

Brinkmann et al. (2003) applied various models, a broken power law (bknpow), a power law plus disk black body $($ pow + diskbb), a power law plus black body $(p o w+b b o d y)$, and a power law plus a bremsstrahlung model (pow + brems) to the first XMM data set, and concluded that while a single power law is rejected, the data could not distinguish between the more complex models such as broken power law, a power law plus a multi black body excess, or a Comptonization model. We adopt here a power law plus a bremsstrahlung model because this model provides some direct indication for the temperature of the soft continuum emission and it provided mostly the best fits. On the other hand, the choice of any other possible model should not significantly affect the results for the narrow absorption features. We fix the absorption column density at the galactic value $\left(3.32 \times 10^{20} \mathrm{~cm}^{-2}\right)$ because extra absorption is not required from the fits. The uncertainties of the parameters are quoted at $1 \sigma$ level.

\subsubsection{The data of the observation in 2000}

The spectra of the November 2000 observation in the $2-10 \mathrm{keV}$ band can be modeled well with a power law plus a $\mathrm{Fe} \mathrm{K} \alpha$ line. When extrapolating the fitted model to the soft energy band (0.3-2 keV), we find the well-known soft excess emission, which we then model with an additional bremsstrahlung component. To account for some flux excess around $0.5 \mathrm{keV}$, we add an additional Gauss line there, but it remains unclear whether this is a genuine source intrinsic line feature or whether it merely reflects the spectral complexity at low energies. The results of the fit to the PN data and to the joint MOS1+MOS2 data are given in Table 1 . Some of the parameters show very large errors, resulting from the limited data quality and the obviously more complex low-energy spectrum. Especially the normalizations and the widths of the lines must 
Table 1. Results from spectral fitting assuming fixed galactic $N_{\mathrm{H}}=3.32 \times 10^{20} \mathrm{~cm}^{-2}$. Standard $1 \sigma$ errors from xspec are given; errors on the Fe line parameters are calculated at a $\delta \chi^{2}=2.7$ confidence level.

\begin{tabular}{|c|c|c|c|c|c|c|}
\hline \multirow[b]{2}{*}{ Component } & \multirow[b]{2}{*}{ parameter } & \multicolumn{2}{|c|}{2000 data } & \multicolumn{3}{|c|}{2001 data } \\
\hline & & $\mathrm{PN}$ & MOS & MOS1 & MOS2 & $\operatorname{MOS} 1+\operatorname{MOS} 2$ \\
\hline \multirow[t]{2}{*}{ pow } & $\Gamma$ & $2.16 \pm 0.05$ & $2.13 \pm 0.04$ & $2.14 \pm 0.04$ & $2.11 \pm 0.04$ & $2.13 \pm 0.02$ \\
\hline & $n_{0}^{\dagger}$ & $1.18 \pm 0.11$ & $2.54 \pm 0.20$ & $2.99 \pm 0.19$ & $2.86 \pm 0.19$ & $2.92 \pm 0.11$ \\
\hline \multirow[t]{3}{*}{ Gauss line } & $E_{1}[\mathrm{keV}]$ & $6.29_{-0.055}^{+0.054}$ & $5.96_{-0.083}^{+0.115}$ & $6.08_{-0.04}^{+0.04}$ & (n.def.) & $6.05_{-1.23}^{+1.09}$ \\
\hline & $\sigma[\mathrm{keV}]$ & $0.001_{-0.001}^{+0.087}$ & 0.001 (n.def.) & $0.002_{-0.002}^{+7.54}$ & (n.def.) & $0.001 \pm 0.25$ \\
\hline & norm $^{b}$ & $4.57_{-1.77}^{+2.28}$ & $1.26_{-3.32}^{+3.73}$ & $7.35_{-3.86}^{+2.042}$ & (n.def.) & $3.04_{-1.49}^{+2.25}$ \\
\hline \multirow[t]{2}{*}{ Bremsstrahlung } & $k T[\mathrm{keV}]$ & $0.264 \pm 0.006$ & $0.290 \pm 0.008$ & $0.217 \pm 0.003$ & $0.259 \pm 0.003$ & $0.239 \pm 0.03$ \\
\hline & norm $^{c}$ & $6.43 \pm 0.49$ & $11.34 \pm 0.08$ & $18.40 \pm 0.94$ & $17.11 \pm 0.92$ & $17.41 \pm 0.61$ \\
\hline \multirow[t]{3}{*}{ Gauss line } & $E_{1}[\mathrm{keV}]$ & $0.51 \pm 0.11$ & $0.51 \pm 0.03$ & $0.52 \pm 0.03$ & $0.53 \pm 0.20$ & $0.529 \pm 0.035$ \\
\hline & $\sigma[\mathrm{keV}]$ & $0.001 \pm 1.4$ & $0.01 \pm 0.52$ & 0.01(n.def.) & $0.0 \pm 104$ & $0.001 \pm 0.46$ \\
\hline & norm $^{a}$ & $4.5 \pm 7.4$ & $24.3 \pm 14$ & 21.0 (n.def.) & $1.19 \pm 16$ & $12.14 \pm 6.63$ \\
\hline$\chi_{\text {red }}^{2} /$ d.o.f. & & $0.863 / 466$ & $1.044 / 393$ & $1.103 / 349$ & $1.29 / 347$ & $1.34 / 700$ \\
\hline
\end{tabular}

$\left.\left.\dagger\left(10^{-3} \mathrm{ph} / \mathrm{keV} / \mathrm{cm}^{2} / \mathrm{s}\right) ;{ }^{a} \times 10^{-5} \mathrm{ph} / \mathrm{cm}^{2} / \mathrm{s}\right) ;{ }^{b} \times 10^{-6} \mathrm{ph} / \mathrm{cm}^{2} / \mathrm{s}\right) ;{ }^{c} \times 10^{-3}$, see definition in xspec manual.

Note: (n.def.) indicates extremely large (or undefined) errors.

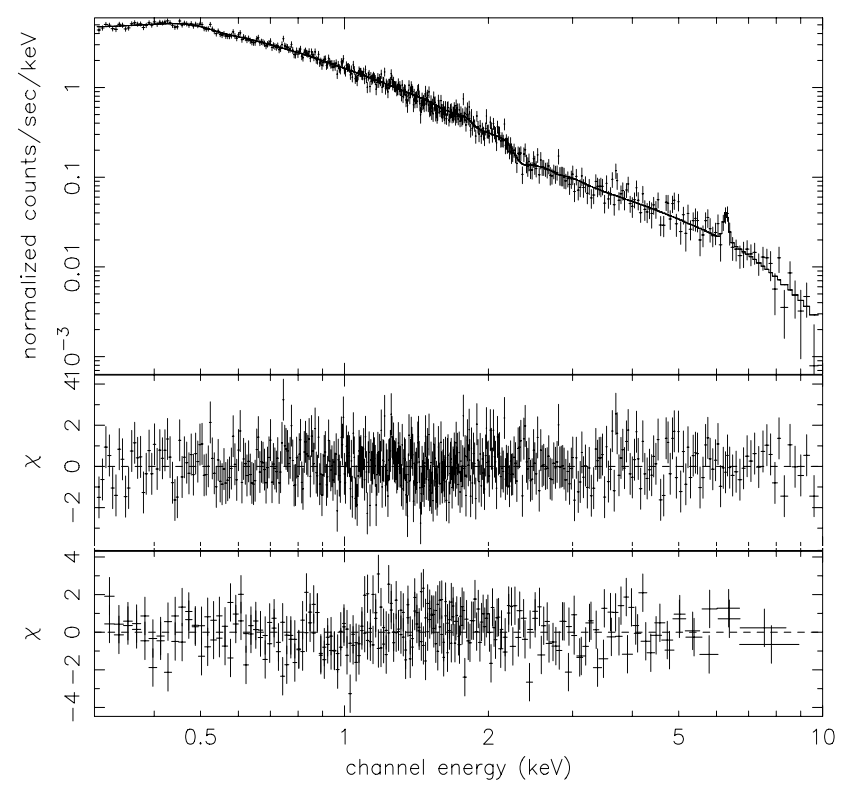

Fig. 1. Fit to the data of 2000 in the $0.3-10 \mathrm{keV}$ band. Upper two panels: PN fit and residuals. Lower panel: residuals from the fit to the joint MOS1+MOS2 data. The MOS residuals are rebinned for clarity.

be used with caution. A significant iron line $(E W \sim 145 \mathrm{eV})$ seems to be present only in the PN data, at $6.29 \pm 0.05 \mathrm{keV}$ (observers frame, corresponding to an ionized line at $\sim 6.7 \mathrm{keV}$ in the source rest frame). From the MOS fit we find $E W \sim 22 \mathrm{eV}$ with very large errors. The residuals of the fits, shown in Fig. 1, suggest that any deviations from the model are relatively small. In particular, there are no apparent absorption lines either at around $8 \mathrm{keV}$ or near $3 \mathrm{keV}$.

Figure 2 shows the PN and the combined MOS fits in the 2.5-3.5 keV energy band. Due to the low effective exposure, the data are rather noisy but the residuals show no clear indications of an absorption structure at $\sim 3 \mathrm{keV}$. Similarly, no

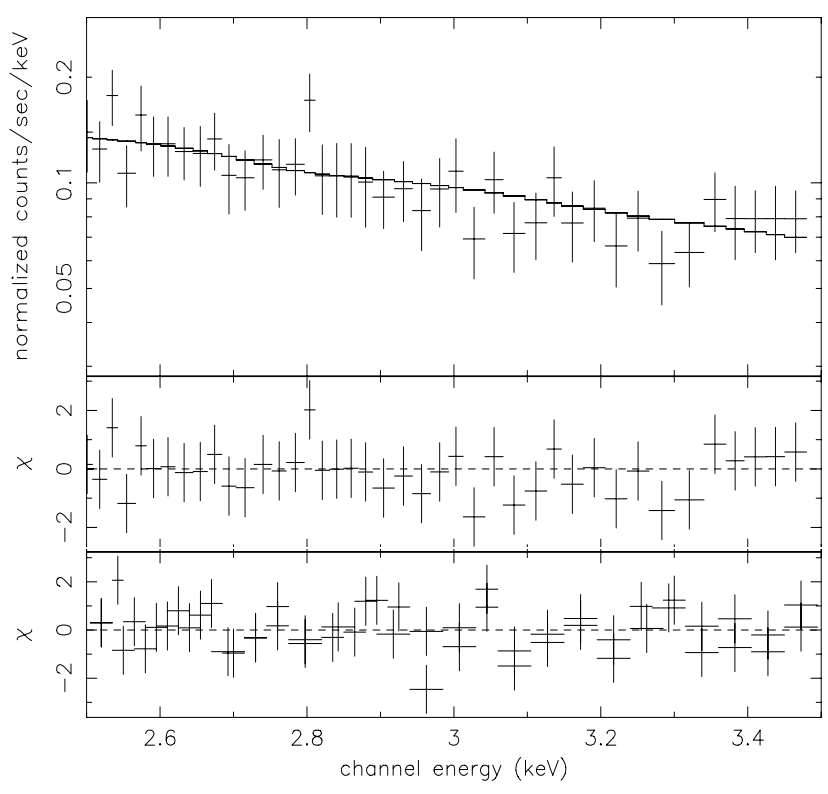

Fig. 2. Fit to the data of 2000 in the $2.5-3.5 \mathrm{keV}$ band. Upper two panels: PN fit and residuals. Lower panel: residuals from the fit to the joint MOS1+MOS2 data.

strong absorption features are visible in the high energy spectra (Fig. 3) at $\sim 8 \mathrm{keV}$, but the signal-to-noise ratio is quite low.

\subsubsection{The 2001 data}

Each of the two MOS spectra of the 2001 observation contains about $\sim 1.09 \times 10^{5}$ counts in the $0.3-10 \mathrm{keV}$ band, thus providing fits with high quality. As above, the $2-10 \mathrm{keV}$ band was first fitted by a model consisting of a power law plus a Gaussian line. Then a bremsstrahlung component was added in the soft energy band. The residuals of the MOS fits were rather noisy and again showed a strong, irregular excess around 


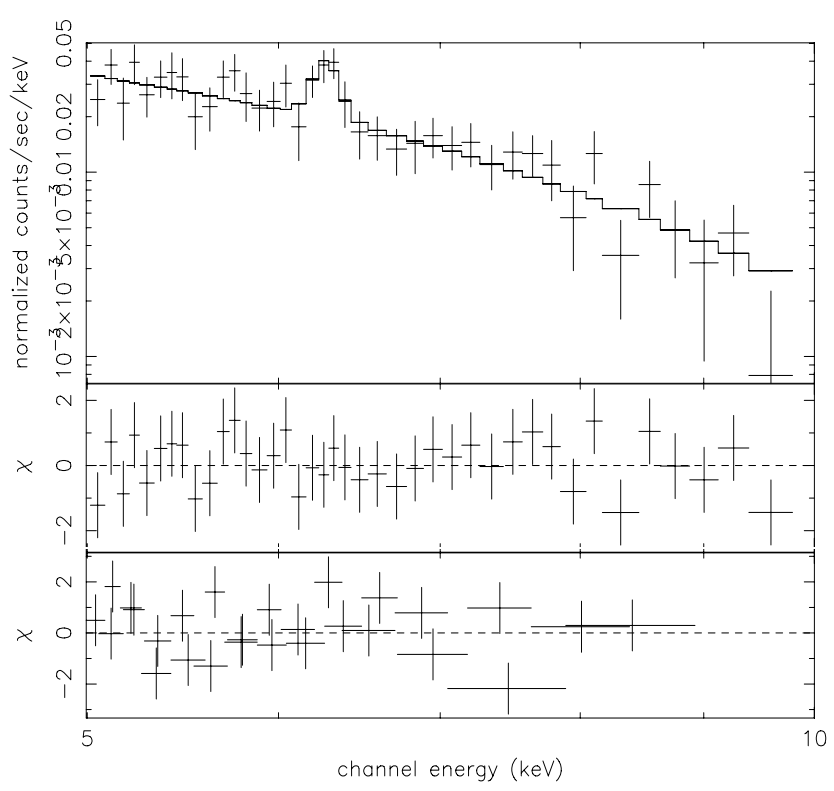

Fig. 3. Fit to the PN data of 2000 in the $5-10 \mathrm{keV}$ band. Upper two panels: fit and residuals. Lower panel: residuals from the fit to the joint MOS1+MOS2 data. There are no good MOS data at energies $\geq 9 \mathrm{keV}$.

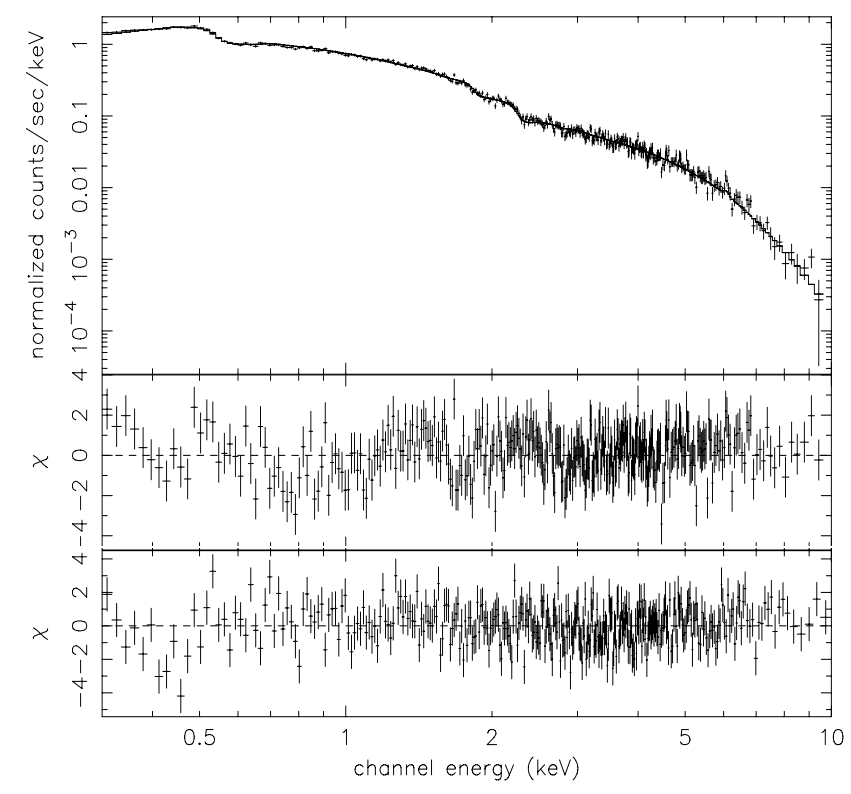

Fig. 4. Fit to the MOS data of 2001 in the $0.3-10 \mathrm{keV}$ band. Upper two panels: MOS1 fit and residuals. Lower panel: residuals of the $\operatorname{MOS} 2$ fit.

$500 \mathrm{eV}$. We therefore added a Gaussian line at these energies, which (for example for the MOS1) improved the fit from $\chi_{\text {red }}^{2}=1.25 / 351$ d.o.f. without line to $\chi_{\text {red }}^{2}=1.10 / 349$ d.o.f. with a line at $E=0.521 \pm 0.03 \mathrm{keV}$. The final fit parameters for the MOS1 and the MOS2 are given in Table 1 and the fit and the residuals are shown in Fig. 4. Table 1 further lists the result of the final joint fit to the two MOS cameras, which is considerably worse than the individual fits due to the scatter of the residuals at low energies.

The fit and the residuals over the $2.5-3.5 \mathrm{keV}$ energy band for the MOS1 data are shown in the two upper panels of Fig. 5,

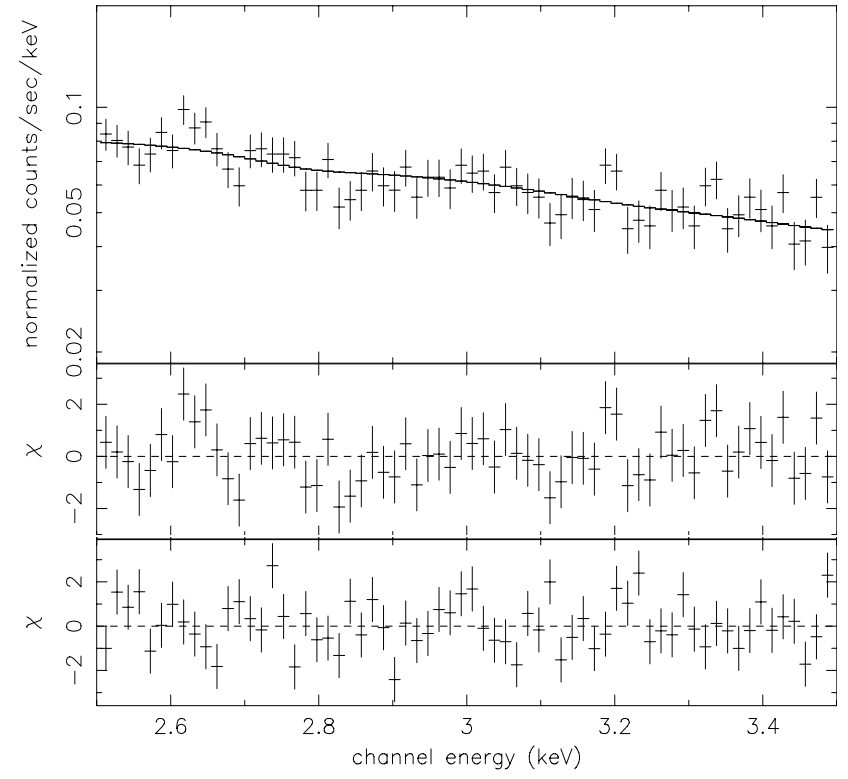

Fig. 5. Fit to the MOS data of 2001 in the $2.5-3.5 \mathrm{keV}$ band. Upper two panels: MOS1 fit and residuals. Lower panel: residuals of the $\operatorname{MOS} 2$ fit.

the residuals for the MOS2 fit in the lower panel. There are no strong residuals seen at $\sim 3.1 \mathrm{keV}$, as claimed by Pounds et al. (2003), despite of the excellent statistical quality of the data with MOS1: 4845 photons and MOS2: 4687 photons in the 2.5-3.5 keV energy band. Fitting a Gaussian absorption line to the MOS1 data, where the residuals show negative values at the appropriate energy, results in a line at $E_{1}=3.116 \pm 0.175 \mathrm{keV}$; the fit gets worse as the total $\chi^{2}$ decreases by $\delta \chi^{2}=0.38$ for 3 additional parameters. To be more quantitative, if we assume a narrow line and an energy resolution of the instrument of $\sim 110 \mathrm{eV}$ (Ehle et al. 2001), we can put a $3 \sigma$ upper limit on the equivalent width of this line of $\sim-31 \mathrm{eV}$. Adding the MOS2 data would argue even more strongly against the physical presence of an absorption line.

Finally, the data in the 5-10 keV band appeared to require an extra emission line near the position of a red-shifted neutral iron line. The improvements over the individual fits with a single power law are marginal, and the line parameters are rather uncertain as there are only about 2100 photons in each MOS in this energy band. In particular, the MOS2 data in the $\sim 6 \mathrm{keV}$ range are so noisy that no definite line position can be obtained from the fits. However, when joining both MOS data sets the achieved accuracy is satisfactory (see last column of Table 1), the improvement of the fit remains small $\left(\delta \chi^{2}=4.4\right.$ for 3 additional parameters), and the equivalent width of the line is $\sim 36 \mathrm{eV}$. The residuals in Fig. 6 further indicate some absorption structure at $\sim 7 \mathrm{keV}$, which can be fitted with a narrow absorption line at $E_{1}=6.98 \pm 0.06 \mathrm{keV}$ with an equivalent width of $E W=-56 \mathrm{eV}$ (with large errors); the improvement in the fit was $\delta \chi^{2}=5.9$ for 3 additional parameters. In the individual MOS data sets this feature appears only as one low energy bin each of width $\delta E \sim 120 \mathrm{eV}$ at slightly different energies. Individual fits with an absorption line yield a worse fit for the $\operatorname{MOS} 1\left(\delta \chi^{2}=0.4\right.$ for 2 additional parameters) and an improved 


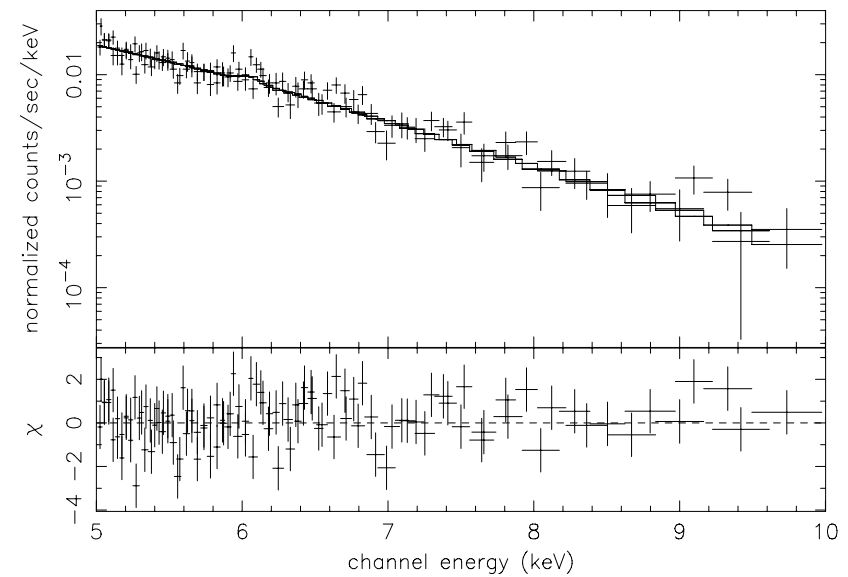

Fig. 6. Fit to the joint MOS data of 2001 in the $5-10 \mathrm{keV}$ band. Note that here the absorption structure at $\sim 7 \mathrm{keV}$ was not fitted.

fit $\left(\delta \chi^{2}=8.6\right)$ for the MOS2. Thus considerably better data are required to assess a reliable confidence level for the existence of an absorption feature.

Again, there are no clear indications of an absorption structure at around $8.2 \mathrm{keV}$ although the low effective area of the MOS detectors at these energies does not allow to obtain very strong upper limits for the existence of absorption line features. The local $3 \sigma$ upper limit for the equivalent width of a narrow line is $\sim-230 \mathrm{eV}$.

\subsection{Temporal variability}

Comparing the spectral fits (see Table 1) for the MOS cameras, we see that the normalization of the hard power law component has only increased slightly between the observations of 2000 and 2001, whereas the power law slope remained the same. The temperature of the soft bremsstrahlung component seemed to have decreased while the normalization increased considerably.

Overall, the average count rate in the $0.3-10 \mathrm{keV}$ band decreased by about $25 \%$ between 2000 and 2001 . Both the hard band flux and the soft X-ray excess varied by nearly the same factor, indicating an unchanging spectral behavior as the total flux decreased. This is an average effect on a time scale of a year. To reveal possible variations on shorter time scales, we generated background-subtracted light curves for both cameras. Because the autocorrelation functions of the individual light curves, as well as the cross-correlation function between the two detector light curves, showed a statistically identical shape with a broad peak at zero lag, we added the MOS1 and MOS2 data to increase the signal-to-noise. We calculated hardness ratios for the long 2001 observation using the count rates in the $0.3-0.8 \mathrm{keV}$ band (soft) and the $1-10 \mathrm{keV}$ band (hard). With this choice both energy bands have similar count rates and the hard band is basically free of the contribution of the soft bremsstrahlung component. The count rates show an amplitude of $35 \%$ variations in the hard band and $38 \%$ in the soft band. The rms fractional variation is $0.116 \pm 0.007$ for both the soft and the hard band. The hardness ratio shows regular variations at small amplitude, which appear not to be strongly correlated with the count rates (Fig. 7).

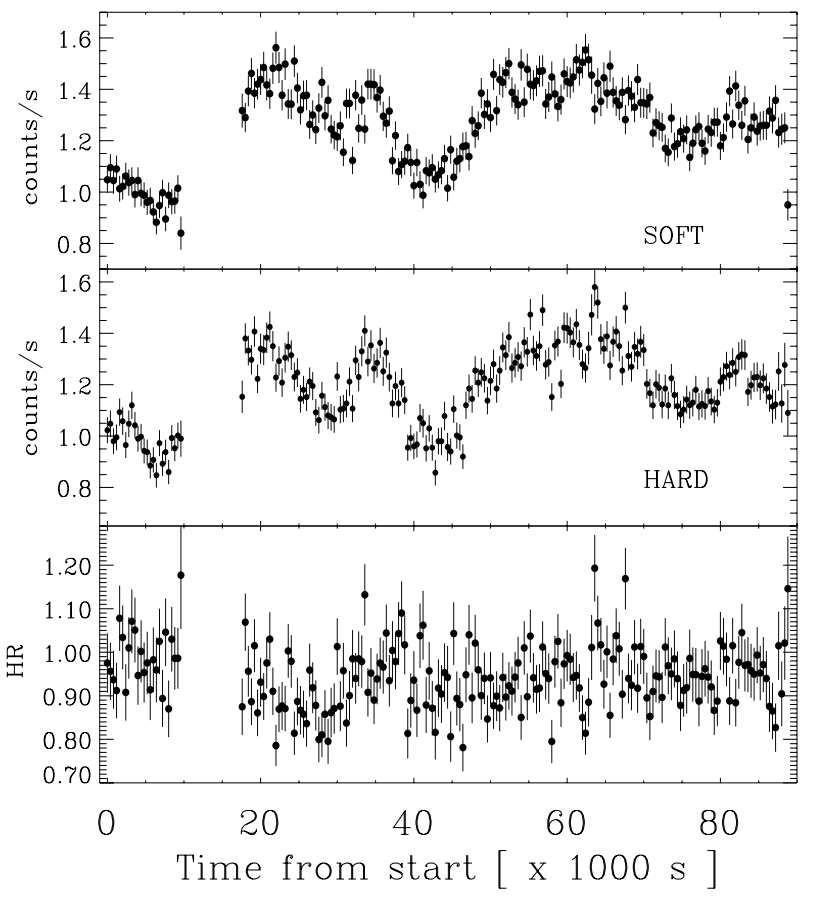

Fig. 7. Summed MOS1 + MOS2 background subtracted light curves in $400 \mathrm{~s}$ time bins; soft band: $0.3-0.8 \mathrm{keV}$; hard band: $1.0-10 \mathrm{keV}$. Bottom panel: hard/soft hardness ratios.

Since the variations in both bands are significant, we checked whether there is a time lag between the hard and the soft X-ray emission. Comparing the light curves of the soft and hard band, it seems that peaks in the soft X-ray light curve lag the hard ones by about a few hundred seconds. However, the cross correlation function (CCF) of the soft versus the hard $\mathrm{X}$-ray light curve displays a flat top shape around lag zero and is slightly skewed at larger lags, but no apparent shift is seen.

This behavior is indicative either of changes of the emission characteristics during the observation interval or of variations of the hard and soft components on different time scales. The observed light curves are characterized by several "events" rising or decaying on different time scales, which suggests that the emission originates from different regions in the system which operate on different time scales. To check this we employed a "sliding window" technique and calculated the CCFs for shorter data intervals, which start at different times of the observed light curve and range over a restricted time interval. Thus, the above-defined $\operatorname{CCF}(\mathrm{k})$ is replaced by a $\operatorname{CCF}(\mathrm{k}$; $\mathcal{T}, \mathcal{L})$; i.e., the cross-correlation coefficient at a lag " $\mathrm{k}$ " is calculated for a data stream with length $\mathcal{L}$ starting at time $\mathcal{T}$ in the light curve. The value of $\mathcal{L}$ can be optimized to match the "characteristic" time scale of the system which reveals best the time evolution of the CCF structure. If the sliding window is very small, only short time correlations between the bands can be identified, because the CCF is dominated by the random fluctuations of the experimental noise; for very long window lengths, local variations of the sign and amplitude of the lags are smeared out and produce cross-correlations with lags around zero, with an asymmetric amplitude distribution (for more details see Brinkmann et al. 2005). 

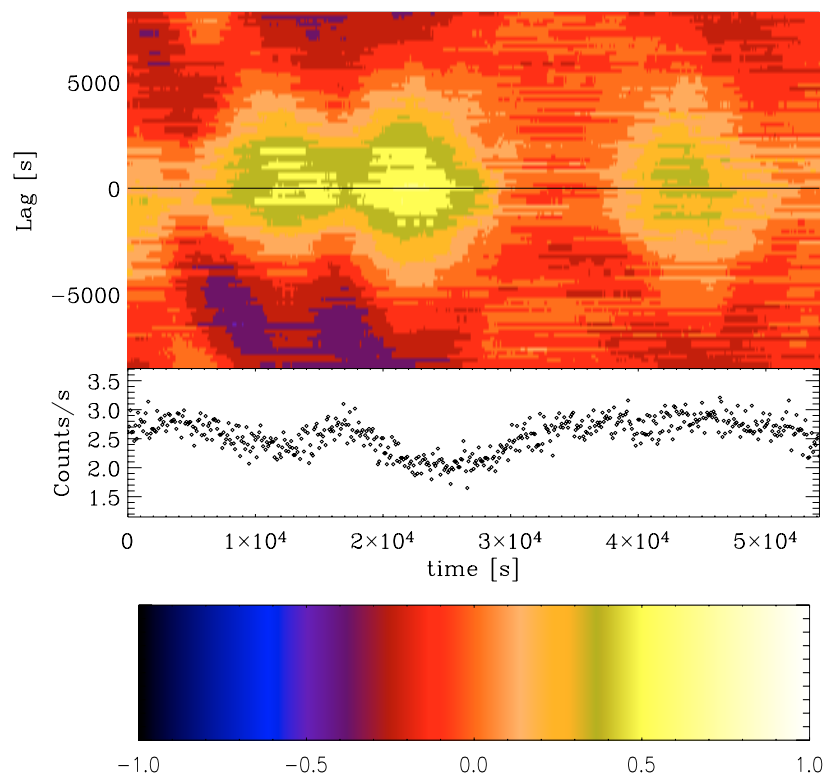

Fig. 8. Sliding window CCF calculated with the optimized sliding window length of $\mathcal{L}=17000 \mathrm{~s}$. The amplitude of the CCF is color coded, with lags plotted in vertical direction. The corresponding light curve in the summed $(0.3-0.8$ plus $1-10 \mathrm{keV})$ energy band is plotted below the CCF. The $x$-axis is the time from the beginning of the second part of the observation (after the gap in Fig. 7) that, for the CCFs, corresponds to the start time of the data window for the calculations. The bottom panel shows the color bar used for the numerical values of the CCFs.

In Fig. 8 we show a two-dimensional representation of this sliding window CCF, based on the above light curves with $100 \mathrm{~s}$ binning. The vertical scale represents the lags, the color coding indicates the amplitude of the cross-correlation coefficient, with the color code given in the lowest panel of the figure. Time is along the $x$-axis. The length of each individual data set is $\mathcal{L}=17000 \mathrm{~s}$. The CCF is not constant during the observation interval. We find three regions where the fluxes are well correlated, obviously related to the flux minima of the light curve, and times at which the fluxes are nearly uncorrelated. On average there seems to be a tendency towards slightly positive lags, however, the data quality does not allow firm conclusions.

\section{Discussion}

The re-analysis of the previous November 2000 XMM observation of PG $0844+349$, and the analysis of a much longer observation in October 2001, does not confirm the presence of high velocity absorption lines at around $3 \mathrm{keV}$ as claimed by Pounds et al. (2003a). Further, neither the re-processed data from November 2000 nor the summed MOS data from 2001 can confirm the presence of an absorption line at $8.18 \mathrm{keV}$.

An analysis of the November 2000 data without the recommended data screening, as done by Pounds et al. (2003a) to increase the signal, introduces spectral distortions due to photon pile up and unpredictable spectral uncertainties from the strongly flaring background. However, even then we cannot confirm the existence of the claimed absorption structures.
There are, however, indications of a weak narrow absorption line at $E_{1}=6.98 \mathrm{keV}$, close to the rest energy of the FeXXVILy $\alpha$ line (6.96 keV) and the FeXXV 1s-2p line $(6.70 \mathrm{keV})$. The physical reality of such a structure can only be assessed by an observation yielding high energy data with considerably higher signal to noise; however, if it is real, this absorption structure can be due to a fortuitous combination of outflow velocity and red-shift in the source, or it might be due to hot local gas. This possibility was pointed out by McKernan et al. (2004) for the identification of the low energy structures found in the RGS spectra of PG $0844+349$ by Pounds et al. (2003b).

The rather different energies, as well as the large errors of the normalizations of an Fe emission line fit, are caused by the inherent noisiness of the data (in particular of the MOS data) in that energy band. From the current data the line position cannot be determined in a robust way. The generally low quality of the fits further strengthens the need for much better high energy data from this source.

Even if an outflow from PG 0844+349 exists, it cannot be as massive and have such high velocities as anticipated from the earlier XMM-Newton analysis. From the numerical simulations of the X-ray spectra of outflows by Sim (2004), one can infer that only models with the lower outflow rates of $\lesssim 1 M_{\odot} \mathrm{yr}^{-1}$ are compatible with the upper limits and/or the weak spectral signals found.

The temporal analysis of the relatively long light curve from the 2001 observation can provide some information on the size of the emission region in the source. Lags on time scales of light-crossing of the hard X-ray emission region are expected in a Comptonization scheme, and are on the order of the light traveling time scale from the hard X-ray emission region to the re-processing zone for re-processing/ionized reflection models for the soft X-ray emission. The models also predict different signs for the lags. Wang \& Brinkmann (2005) found a time lag between hard and soft X-rays on the order of $10^{3} \mathrm{~s}$ in RX J0437.4-4711, in support of a Comptonization picture. For the Fe K $\alpha$ profile of MCG 6-30-15, Fabian \& Vaughan (2003) argued that hard X-rays must be emitted mainly within a few gravitational radius. Thus, a time lag should be on the order of $10^{3} \mathrm{~s} \times\left(M_{\mathrm{BH}} / 10^{8} M_{\odot}\right)$ in Comptonization models. Peterson et al. (2004) estimate a black hole mass of $(0.9 \pm$ $0.4) \times 10^{8} M_{\odot}$ for PG $0844+349$. Thus the hard X-rays in PG $0844+349$ should lag the soft by a few $10^{2} \mathrm{~s}$ to $1 \mathrm{ks}$. Due to the flatness of the $\mathrm{CCF}$, time lags on the order of a few hundred seconds cannot be addressed, but the CCF is consistent with a shift in the peak position of this time scale. The skewness and the changing behavior of the CCF indicates, however, that there might be more than one emission region contributing to the observed flux.

\section{Conclusions}

We have analyzed $80 \mathrm{ks}$ MOS data from a serendipitous observation of PG $0844+349$ and the re-processed EPIC data from the November 2000 observation of the source. We cannot confirm the strong spectral signatures taken by Pounds et al. (2003b) as evidence for high velocity outflow from the source. 
The nature of the absorption structure at $\sim 7 \mathrm{keV}$ remains obscure as do the position and equivalent width of the red-shifted iron line in the two observations. The position of the fitted $\sim 0.5 \mathrm{keV}$ line is indicative of OVII in emission. However, such features are frequently found in MOS/PN spectra and might be caused by an incorrect soft emission model and/or remaining uncertainties of the detector calibrations. A cross-correlation analysis of the soft and hard light curves does not yield any clear evidence for temporal lags, due to the limited statistical quality of the data. Thus only data with considerably better quality can reliably answer the question of the physical conditions in the center of this narrow-line Seyfert galaxy.

Acknowledgements. This work is based on observations with XMM-Newton, an ESA science mission with instruments and contributions directly funded by ESA Member States and the USA (NASA). TGW acknowledges financial support from the MPG - Academia Sinica exchange program and thanks MPE for hospitality where part of the research was done.

\section{References}

Aschenbach, B. 2001, Proc. SPIE, 4496, 8

Brinkmann, W., Branduardy Raymond, G., Grupe, D., \& Ferrero, E. 2003, A\&A, 398, 81

Brinkmann, W., Papadakis, I. E., Raeth, C., Mimica, P., \& Haberl, F. 2005, A\&A, 443, 397
Chartas, G., Brandt, W. N., Gallagher, S. C., \& Garmire, G. 2002, ApJ, 579,169

Chartas, G., Brandt, W. N., \& Gallagher, S. C. 2003, ApJ, 595, 85

Dasgupta, S., Rao, A. R., Dewangan, G. C., \& Agrawal, V. K. 2004, ApJ, 618, L87

Ehle, M., Breitfellner, M., Dahlem, M., et al. 2001, XMM-Newton Users' Handbook, http://xmm.vilspa.esa.es/xmm_user_support/ external/documentation/uhb_frame.shtml

Fabian, A. C., \& Vaughan, S. 2003, MNRAS, 340, L28

Hasinger, G., Schartel, N., \& Komossa, S. 2002, ApJ, 573, L77

Kaspi, S., \& Behar, E. 2005, ApJ, accepted [arXiv: astro-ph/0509562]

King, A. R., \& Pounds, K. A. 2003, MNRAS, 345, 657

Leighly, K. M., Mushotzky, R. F., Nandra, K., \& Forster, K. 1997, ApJ, 489, L25

McKernan, B., Yakoop, T., \& Reynolds, C. S. 2004, ApJ, 617, 232

Peterson, B. M., Ferrarese, L., Gilbert, K. M., et al. 2004, ApJ, 613, 682

Pounds, K. A., Reeves, J. N., King, A. R., et al. 2003a, MNRAS, 345, 705

Pounds, K. A., King, A. R., Page, K. L., \& O`Brien, P. T. 2003b, MNRAS, 346, 1025

Sim, S. A. 2005, MNRAS, 356, 531

Titarchuk, L. 1994, ApJ, 489, 570

Vikhlinin, A., McNamara, B. R., Forman, W., et al. 1998, ApJ, 502, 558

Wang, T., \& Brinkmann, W. 2005, A\&A, submitted 